\title{
Electron nanocrystallography - solving small crystal structures by TED.
}

\author{
J.C.H.Spence and J.S. Wu* \\ Department of Materials, Cambridge University, Cambridge, UK. CB2 3QZ. (Spence@asu.edu) \\ *Department of Physics, Arizona State University, Tempe, Az. 85287. USA.
}

The early part of John Cowley's career (from 1947) was devoted to finding unique applications for electron crystallography, and to quantifying transmission electron diffraction (TED) patterns to solve crystal structures [1]. The hope than (and now) was that nano-crystals, too small to be solved by X-rays, could be solved by electron microdiffraction, and that use could be made of the greater sensitivity of electron diffraction to ionicity and hydrogen. Despite some successes in Australia and Russia, the instrumentation available at that time did not allow routine methods to be developed for doing this, and his interests moved on to diffuse scattering, dynamical forbidden reflections, ordering in alloys, Fourier images and the multislice (with A. Moodie).

Two things have changed since then : Today's instruments offer huge advantages over those of the nineteen-fifties. Automated tomographic imaging in biology (more recently in materials science) can surely now be applied to the automated collection of 3D diffraction data from nanocrystals. (Experience with direct and other phasing techniques shows how vital are 3D, rather than 2D, data sets, together with atomic resolution diffraction data). Other instrumentation developments include field-emitters, electronic detectors, cold stages, better vacuum, computer control and plasma cleaning. But it is the in-column elastic imaging energy filter which has been the greatest recent contribution to the quantification of electron diffraction data. Scripted computer control raises the possibility of sample position adjustment by fiducialising after tilting to correct for motion during tilt. Secondly, the boom in nanoscience has created a huge demand for a routine methods for solving the host of new light-element inorganic nanostructures which are being created. Their crystal size allows use of kinematic theory, and hence numerical methods for phasing. HREM may also be combined with TED to help solve layer-strucutres [2]. The development of the modern precession camera, minimizing dynamical effects (as suggested by Blackman), has been important [3].

Since multiple scattering and poor quality of data (due to sample imperfections) has dogged this field from the beginning [4], we have focussed on structure-independent experimental tests for single scattering conditions, and computational solutions to the phase problem which work with electron diffraction data. A test of various kinematic approximations and extinction models compared with elastically filtered experimental data can be found in [5]. For a thin crystal which is known to be noncentrosymmetric in projection, the appearance in thin regions of a centrosymmetric diffraction pattern is a strong indication of single-scattering conditions. Secondly, we use a "blank disk" CBED test [6] - the appearance of CBED disks in thin areas with little modulation also strongly suggests single scattering, as shown in figure 1 for Anthracene at liquid helium temperature. (Recall that choosing one pixel from each CBED disk gives a complete TED spot pattern from a very small area, unlikely to be defective or bent). Note that absent forbidden reflections (in perfect-crystal regions) do not indicate single scattering [7]. A strong direct beam is a good indication, as is weak contrast in HREM images. CBED patterns for space-group determination, and EELS and EDX data for composition clearly help also. The Bravais lattice can be found using inner HOLZ lines [8], and the most difficult unknown quantity needed to solve a nano-structure may be its density. 
While modified X-ray direct methods can certainly be applied to atomic-resolution kinematic electron diffraction data to solve the phase problem [4], we have found that the new "flipping" algorithm of Ozlanyi et al is quite remarkably successful and simple [9]. It does not required atomic-resolution data. We have applied it successfully to X-ray diffraction data from unknown structures [10] and are now applying it to electron and X-ray data from crystalline and non-periodic samples [11] for diffractive imaging [12]. All of this electron crystallography work is greatly facilitated by the Univ of Illinois Web eMaps software, which runs mostly in real-time on the web. It draws structures, finds structure factors, indexes patterns and simulates HREM, CBED and TED patterns, etc. [13].

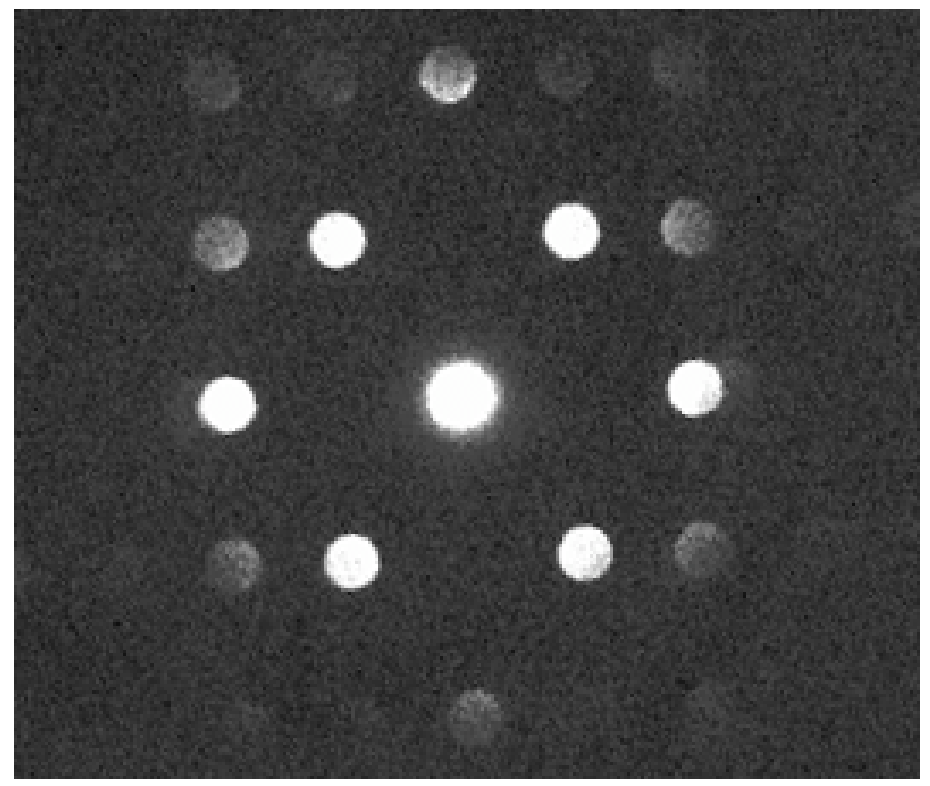

Figure 1. "Blank disk" CBED pattern obtained at $25 \mathrm{~K}$ to reduce radiation damage from Anthracene using the in-column elastic imaging energy filter of the Leo 912 and a liquid helium double-tilt holder.

References

[1] J. M. Cowley. Crystal structure determination by electron diffraction. In Progress in Materials Science. Vol 13, p. 269 (1967) Pergamon Press (Oxford).

[2] X. Zou, Z.Mo, S. Hovmuller, X. Li and K. Kuo. Acta Cryst A59,(2003) p.526

[3] R.Vincent and P. Midgely. Ultramic. 53, (1994) 271

[4] D. Dorset. Structural electron crystallography. Plenum. (1995). New York.

[5] J. Wu and J. Spence. Micros and Miconan. 9, (2003) p.428.

[6] J. Wu and J. Spence. Acta Cryst. A58, (2002) p. 580

[7] J.C.H.Spence and J.M.Zuo. Electron Microdiffraction. (1992). Plenum. New York.

[8] J. Zuo. Ultramic. 52, (1993) p. 459

[ 9] G. Oszlanyi and A. Suto. Acta A. (2005). In press. And Acta A60, (2004) p. 134

[10 ] J. Wu, J. Spence, M.O'Keeffe, T.Groy . Acta Cryst. A60, (2004) p. 326

[11] J. Wu and J. Spence. Acta A. (2005) In press.

[12] J. Zuo et al. Science 300, (2003) 1419.

[13] Web emaps: http://emaps.mrl.uiuc.edu/ Thanks to EPSRC and ARO. 